\title{
EL “LLIBRE DE CAPITOLS AB LOS QUALS SE ARRENDEN Y COLLECTEN LOS DRETS REALS QUE TE SA MAGESTAT EN LA GOVERNACIO Y BATLIA GENERAL DE ORIOLA Y ALACANT» DE LUIS OCAÑA
}

\author{
Felipe Mateu y Llopis \\ Catedrático de Universidad
}

\section{I.-DRET REAL DE ALMOXARIF}

Escasamente utilizado, por su rareza, es el libro cuyo título y portada completo es Llibre de Capitols ab los quals se arrenden y Collecten los drets Reals que te Sa Majestat en la Governacio y Batlia general de Oriola y Alacant, ab les declaracions de com se executen y practiquen. Fetes per lo Doctor Luis de Ocaña Cavaller, Assessor per sa Magestat de dita Batiia general, precehint decret y manament de sa Magesta ab una Real lletra. Ab llicencia del Ordinari. Impres en Oriola per Augusti Martinez. A despesses de sa Majestat. Any 1613.

De esta obra se dio un comentario en Saitabi, XXXI (1981), bajo el título loanna Infanta de las Españas, Princesa de Portugal y la Baylia general de Oriola, desde el punto de vista diplomático, de esta intitulación. Trátase aquí ahora, correspondiendo a la invitación de doña María Luisa Cabanes Catalá, secretaria de la revista Anales de la Universidad de Alicante, Historia Medieval, de la Facultad de Filosofía y Letras de la Universidad de Alicante, de extender los comentarios a la citada obra, analizando los drets a que se refiere, de tanto interés para la historia de aquella Gobernación; evitando las notas de pie de página, se citan los folios del impreso, que es, por otra parte, un texto riquísimo para diversos estudios, como el de la lengua, el derecho, la administración y varios más.

Se mencionan, por este orden, en la Carta del Autor al rey, "los 
drechos Reales de Almoxariffe, Aduana, Vedado, Servicio y Montazgo, Estremeño, drechos de Peso y saca de la sal de la Mata", de los que se dará comentario aquí, y de los que da relación más extensa la Taula dels drets Reals que es arrenden en la present Batlia general de Oriola y Alacant. Dret Real de Almoxarif. Dret Real del Vedat. Dret Real de Servicio, y Montazgo. Dret R. del Estremeño, o de mig Delme. Dret Real de Pastura. Dret Real de Pesos de Peix. Dret Real de Aduana. Dret Real de saca y venda de la sal de les Salines de la Mata.

De esta obra existe un ejemplar en la Biblioteca Universitaria de Valencia y otro en la Biblioteca de Catalunya, con el ex-libris de Nicolas Pastor y Miravete, que ha sido el utilizado aquí.

El Diccionario de Historia de España (1968) da referencias del Almojarifazgo en Castilla; había concedido Fernando III este derecho a SeviIla; las aduanas del Almojarifazgo rendían este impuesto administrado por arrendamiento. Pero no se citan otras ciudades que en el Llibre de Ocaña se mencionan, como Cartagena y Murcia. Vicente Martínez Morellá dio en Privilegios y franquezas de Alfonso $X$ el Sabio a Alicante (1951); en 15-I-1256 Alfonso $X$ a "Berenguel de Moncada o a quien quiere que sea el amoxarife de Murcia mandando que no exija portazgo a los de Alicant -así se escribía el topónimo.

El rey había dado en 5-X-1252 al Conseijo de Alicant el Fuero de Córdova; en 11-VII-1257 disponía que si mercadero o otro ome estranio cualquier vendiere cosas menudas en Alicant que non de ende drecho alguno al mio almoxarifazgo de Alicant; y en 16-IX-1261 otorgaba que los mercaderes estranios que mercaduras troxieren por mar o por tierra a Alicant et las vendieren en la villa que paguen al almojarifazgo de Alicant quatro moravedis por centenar et non den otro drecho ninguno por razon del dieçmo ni de almoxarifazgo, con referencias al de Murcia. En 3-V-1272 Sancho IV daba privilegio sobre la feria de Orihuela y en $30-V-1280$ confirmaba a la villa los Privilegios dados por sus antecesores; se citó en el Catalogo de la Exposición de Derecho Histórico del Reino de Valencia (1955).

En 1954 Vicente Martínez Morellá publicó Cartas de los Reyes de Castilla a Orihuela. 1265-1295, en cuyo prólogo menciona la obra de Pedro Bellot, oriolano, Compendio de lo que se contiene en las notas antiguas de la Sala de la Ciudad de Orihuela hasta el año 1523 con un tratado de la Germania y otro de los bandos y desafios, transcrito por Juan Torres Fontes con un estudio preliminar.

Aquellos años de la Germanía han sido estudiados por María de la Eucaristía (María Luisa Chiarri Martín) en el libro Orihuela y la guerra 
de las Germanias publicado por la C. de A. y S. y M. de P. de Na Señora de Monserrate en 1963.

En 3-Il-1296 don Alfonso el de la Cerda notificaba al Concejo y hombres buenos de Orihuela haber dado el reino de Murcia al rey de Aragón. En la Bibliografia de la Diócesis de Orihuela publicada por don Isidro Albert Berenguer, en 1957, se cita la nueva edición hecha en Orihuela en 1791 por el doctor don Luis de Santa Cruz, de las Ordenanzas y Reales Estatutos para el buen govierno y recta administración de los caudales del Tercio-Diezmo formados por don Luis de Ocaña, para suplir la notable falta de ejemplares antiguos".

El mismo V. Martínez Morellá dio en 1951 Privilegios y franquezas de Alfonso $X$ el Sabio a Orihuela; en el de 20-VII-1271 consta la intitulación Nos don Alfonso por la gracia de Dios, Rey de Castiella, de Toledo, de Leon, de Gallizia, de Sevilla, de Cordova, de Murcia, de Jahen et del Algarve, en uno con la Reyna dona Violant nuestros ffijos el inffante don Fernando primero el heredero et con don Sancho et con don Pedro et don Juhan et don Jaymes; en la cláusula de soberanía añadía a aquellos reinos de las ciudades de Baeça y Badalloz; en el de 11-VII-1257 se cita el almoxarifazgo de Alicant, como en el de 1-V-1278, y en 15-I1256 el de Murcia; en $1260-30$ junio- el rey en hallaba en Cordoba; en marzo de 1275 actuaban los almoxarifazgos de Alicant et Orihuela, habiendo pleito entre el Conceijo de ésta y el almoxerif.

Ya en 1915 Abelardo Merino en su Geografia histórica del territorio de la actual Provincia de Murcia desde la Reconquista por D. Jaime I de Aragón hasta la época presente, tratando de la hacienda escribió que "a los Almoxarifes de Alfonso X suceden los Contadores" y las resistencias a pagar los tributos.

En cuanto a la especie de moneda con que se pagaba el almoxarif en Murcia, escribe Juan Torres Fontes, en La ceca murciana en el reinado de Alfonso X (A. A. el Sabio, 1957): "Cuando en el año 1250 Alfonso $X$ hubo de dotar a la Sede Cartaginense que se restauraba, le concedió una buena parte de los tributos que se había obligado a entregarle el rey moro de Murcia en la capitulación de Alcaraz, en que se especificaba que el monarca de Castilla cobraría la mitad de las rentas reales pertenecientes al reyezuelo musulmán. La dotación fue de diez mil morabetines, y así especificaba: "annuatim decem milia aureorum usualis monetae»; de ellos cinco mil en las rentas de Murcia ("de reditibus Murtiae quinque milia aureorum... de reditibus Elche mille... de reditibus de Oriola duo milia... de reditibus Lorca duo milia aureorum", maravedis, que eran la moneda de oro acuñada "en la ceca de Murcia bajo Muhammad Abenhud Bahaodaula»; en Aledo, en cambio, se 
pagaban las rentas del amojarifazgo en 1271 en maravedis alfonsis, mandados acuñar según el Ordenamiento de Posturas de Jerez de la Frontera, en 1268, como consta también por carta del rey de 1272: "mandé fazer moneda de maravedis de oro fino que an de la una parte castello et de la otra leon"; en 1258, año $656 \mathrm{H}$. se acuñaban en Murcia semidoblas y cuartos de dobla.

El reino de Valencia constituido por Jaime I (1238-1276) tuvo una muy temprana división político-administrativa, dada su extensión en sentido de los meridianos y escasa anchura en el de los paralelos, sirviendo los ríos de hitos divisorios; así, hacia el sur, con densísima población de sarraceni, ejercia autoridad el Locumtenens procuratoris in regno Valencie ultra Xúcharum, que en 1283 era Eximeno Çapata; en 1286 Pedro de Libiano era el Thesaurarius ante quien redituaban las aljamas sarracenorum, en aquel siglo de vida mudéjar, estudiado por Francisco A. Roca Traver, en 1952. Recientemente Robert I. Burns en las "Actas de I Simposio Internacional de Mudejarismo" (1981) ha dado precisos documentos sobre "el mushrif y el título de móixerif de los años posteriores", 1286.

Alfonso III de Aragón (1285-1291) nombró a un pariente de aquél, Pedro, llamado Bernard de Llibià, almoxeriffus montanearum nostrarum ultra Xucharum; en 1290 había un almoxerifatus montanearum nostrarum Regni Valencie ultra Xucarum, el amojarifazgo, propio de los reinos conquistados por Fernando III (1217-1252), su hijo Alfonso $X$ (1252-1284) y el suegro de éste, Jaime I (1213-1276), repartidos entonces, entre Castilla y Aragón. Sin duda alguna el moixerif de las comunidades moras era el recaudador de tributos y cuando en el Reino de Valencia se integraron las tierras de Orihuela, en la nueva demarcación ultra Xixonam, el almoxarif, cuya legislación estudió Luis de Ocaña, en 1613 tenía unos antecedentes que lo habian convertido ya en derecho consuetudinario, el cristiano, que los tenía a su vez en el sarraceno.

Recuérdese que el infante don Alfonso de la Cerda, para ganarse la ayuda de Aragón, había hecho donación del reino de Murcia a Alfonso III en 26-VI-1289 y que más tarde, Jaime II haría lo mismo en 21-I-1296 y el Consejo de Orihuela le rendiría homenaje en $11-\mathrm{V}$ de aquel año; recuérdese también la presencia de Jaime ll en el malogrado sitio de Almeria de 1309 y que la idea de que pasara Murcia a la corona aragonesa persistió durante Pedro IV (1336-1387) y Enrique II de Trastámara (1369-1379) cuando éste luchaba contra su hermano Pedro I (1350$1369)$.

Ocaña aclaraba que el privilegio de Jaime II de 1321 , folio $L X X$ v. del número CXXVIII, del Aureum Opus, no s-enten del Almoxarif de 
Oriola, y que la concesión de franqueza por leuda y peatge no-s pot estendre al dret Real del Almoxarif, el de Oriola (75, r. 76 r.).

Recuerda Ocaña que en el Llibre de visita que-s fa en lo any 1483 recondit en lo offici de muestre racional de la ciutat y regne de Valencia se troba de que les robes y mercaduries que van de pas, se deu pagar dit dret Real de Almoxarif als Collectors y Arrendadors.

De especial interés es la provisión de Fernando ll de 29 de julio de 1493 dada en Barcelona, sobre que los estrangers no sien tranchs, dirigida a don Diego de Torres Batle general, del Regne de Valencia, sobre quienes se excusaban del pago del dret de peatge è leuda.

Del dret de peatge, del medieval pedáticum, amplia información en el Catálogo de la Exposición de Derecho Histórico del Reino de Valencia, serie de volúmenes en el Archivo del mismo Reino, en la Sección del Maestre Racional, ante quien pasaban las cuentas de su administración, así como también del dret o impuesto de la leuda, lleuda o lezda, gravamen por el paso de géneros por los puertos o fronteras.

En los Fori antiqui Valentiae, Rúbrica CXLIV, de lezda, hostalagio, corredoribus, se hallaba establecido que los habitadores et populatores civitatis Valentiae et eius termini non donent unquam pensum, mensuraticum, lezdam, pedaticum, vel ribaticum de aliquis mercibus, de lo que estaban libri et inmunes a predictis omnibus in civitate Valentiae et toto termino eiusdem civitatis; era en 22-X|-1239.

En Aragón lezda; en el Fuero de Calatayud, Nullo vicion de Calatayub qui pasarat por los portos de Pampilona vel per ipsos de Jaca non donet lezda in ida neque in venida; lo estudió Manuel Alvar en El peaje de Jaca de 1437, en los "Estudios dedicados a Menéndez Pidal», II, 92-333.

En folio $21 \mathrm{r}$. del Llibre de Ocaña se hace referencia al Almoxarifazgo de Granada, libro 9 de la Nueva Recopilación; en folio $22 \mathrm{v}$. al Llibre de visita ques feu en lo any 1488 recondit en lo offici de mestre racional de la ciutat y regne de Valencia se troba de que les robes, y mercaduries que van de pas, se deu pagar dit dret Real de Almoxarif als Collectors y Arrendadors en dita ciutat, aduciendo que en la disposición de 8 de enero de 1504 no y ha capitol de ques collecte de robes y mercaduries que van de pas, y por dicha causa no se cobrara; habia litigios sobre dicho pago en ocasiones del comercio con Murcia; se arrendaba lo dret Real de Duana en ciutat de Alacant.

Ocaña definía que Lo dret de Moxarif se deu pagar en lo lloch hon està la cosa venuda è comprada, o aparaulada, y no es te compte del Iloch del contracte $(46, v$.) y en cuanto a la infracción dice: Esta pena de 
perdició y confiscació imposada contra los fraudants del dret Real de Almoxarif, es conforme al dret comu dels Romans, aduciendo textos de juristas $(48, v$.).

El vectigal, vectigalis, se halla en Claudio, ferre vectigal, pagar el tributo; vectigal agro imponere, en Cicerón; vectigali levare, en el mismo; en Salustio vectigalium y en Suetonio Vectigaliorum: Nuestros tratadistas, del XVI y XVII, como Ocaña, eran romanistas, que se basaban en leyes, como la lex Claudia, de disposiciones tan antiguas, de 224 y 119 a. J. C. sobre possessores y proprietarii del ager publicus, imponiendo o levantando el vectigal quedando vedada a los del estado senatorial la recaudación de los pública vectigalia. $Y$ todavía Matheu y Sanz en su Tractatus De Regimine Regni Valentiae (ed. 1704, 91) empleará la palabra diciendo: Vectigalia impósita in comitiis et quid imponantur; recordaba que Vectigalia sibi Regnum imponit ad solvenda donativa curiarum, como en las de Alfonso III, el V de Aragón, de 14171418, a las que se refería la disposición de Philippus Primogenitus Caroli Quinti Imperatoris Rex Hispaniarum, en las de Monzón de 1547.

En el folio 1 comienzan los Capitols ab los quals se arrenda y colecta lo dret Real del Moxarif de la ciutat de Oriola, e de la Vall de Elda, diciendo: Deu exigir è rebre dit collector, o Arrendador de qualsevol mercaderies se venen è compren en la ciutat de Oriola, e son terme un sou per lliura del comprador; esto es, un sueldo de 12 dineros, por libra de 20 sueldos, moneda valenciana; se mencionan los casos de qualsevol que tindra franquea; fueron dados los Capitols, Dilluns contant vuit dies del mes de laner, del any de la Nativitat de nostre Senyor 1504, de manament del molt maginifich mossen Pere Desprats Cavaller Llochtinent de Batle general, per instancia e requesta del honorable y discret en Nicolau Morelles Notari Arrendador del dret en lo present any; en aquel año murió Isabel de Castilla, quedando Fernando II como rey de Aragón con este ordinal.

En el folio 6 se decía: A inteligencia y declaració del primer Capitol, fa advertir que Almoxarif es vocable Arabich, y significa en nostron parlar y es lo mateix que Colector de drets y rentes Reals com o diu la l. tit. 9 p. 2 etc. ibi. Greg. López y Montalvo, remitiendo a la Nueva Recopilación y los citados y añadiendo: $y$ asi la exactio de aquest dret te principi de temps que España era de Moros; repite la cita de Montalvo y López y sigue así: y per ara es Colecta dit dret Real de Moxarif, y se ha de pagar per tots aquells que no tinguen franquea, a raho de un sou per iliura lo venedor y altre sou lo comprador, y lo mateix es dispost en lo Capitol, 9, infra eodem, e açó si son Christians. Pero si son Moros, lueus, o altres infeels los tals dehuen pagar vint diners per lliura, ço es 
lo venedor, vint dines y lo comprador altres vint dines, si los dos son infeels segons se troba en la declaracio antiga del primer Capitol de dit dret.

Se declaraba que los qui porten forments, moltons, cabrons, cabres, bous, $y$ porchs vius a vendre a la present ciutat y Regne, son franchs de tots dret reals y que las citadas mercaderías no necesitaban del albaran del Desmero de Castella, constando que en temps del Rey don Fernando 102 . se arrendava 10 dret Real de Almoxarif, en les viles de Elig, y Crevillent, como se arrendava en Oriola, $y$ en tote la Vall de Elda; les viles de Elig, Crevillent, Asp, Novelda y totes les demes de la Vall de Elda, son del districte de Oriola.

Expone Ocaña cómo no es exegeix ni cobra ni es degut dit dret de les coses ques compren per lo proveidor de les armades de sa Magestat de son orde Per provisio y bastiment de la armada $(23, \mathrm{v}$.); trata de las excepciones de lo Bisbe, o Pabordre y Capitol, recordando el fur 245 de Felipe II, de 1585; el Llibre es un diplomatario, en gran parte, por insertar cartas reales dirigidas a los Virreyes; una de 1554 al Marqués de Aytona; se hallaban exentos también los portadores del forment que de Castella, o altra qualsevol part axi del present Regne, com per mar, es portarà a vendre a dita present ciutat e son terme (30, v.-31 r.); igualmente lo estaban los caballeros de Montesa, Santiago, Alcántara - Calatrava, citándose privilegio del Rey En Pere el 2-Pedro IV de Aragón, con mención de tratadistas como Lazarte, Michael Ferrer, Fontanella y otros sobre gabelas; y una provision real de Carlos I de 1549 registrata in comuni Valentiae, 24 fol. 20, la qual esta insertada originalment en lo primer llibre de lletres y provisions Reals recondit en lo Archiu de la Ciutat de Oriola, a cartes 358 (33 v.).

En 30-VIII-1555 se ordenó que las causas, asi en la primera instancia como en la de appellacion, se restituyessen a la Batlia general della Xixona, que es la de Alicante y Origuela $(80, v$.). Recuerda Luis de Ocaña que Oriola fonch erigida en Cathedral, en lo any $1566(105, v$.). Sobre la erección de la Catedral orcelitana puede verse fácilmente $E I$ primer Sínodo de Orihuela. 1569, publicado por José María Navarro Botella (1979) y Catalogación y extracto de fondos de los pergaminos existentes en el Archivo de la S. I. Catedral de la Ciudad de Orihuela (I.E.A. 1977).

Especifica el Llibre de Ocaña los casos en que uno sia franch y exempte de pagar drets Reals en los 15 dies de la fira y que lo dia de mercat y fira no es pot tenir en dia de festa.

En el segundo Capitulo trata de aquellos quince dias de la feria que 
comenzaban en el de Todos Santos, durante los cuales no se debía cobrar drets Reals de Almoxarif y Quema, por sentencia donada in contradictorio judicio en lo supremo Real Consell de Arago entre parts, de una lo Sindich de la Ciutat de Oriola, y de altra lo Procurador fiscal de sa Magestat, de 17 octubre de 1567 (39).

El Baile estaba obligado a nombrar colector: Lo dret de Moxarif, se deu pagar en lo lloch hon està la cosa venuda è comprada, o aparaulada, y no es te compte del lloch del contracte; se insiste (46 v.). Se especifica el caso de lo vehi que rescatara algun esclau o catiu Moro, o Mora, y lo tal s-en anirà fora ciutat, ò a embarcar, sens manifestar o pagar lo dret del preu del tal rescat, debiendo dicho derecho el amo o comprador (53).

Aduce la disposición de 13 de julio de 1572 sobre casos de avecinamiento; se insertaban en lo llibre, de lletres y provisions Reals, fol. 26 recondit en lo Archiu de la Batlia general de Oriola, que reproduce, como otras de 30 de junio de 1573, y 14 de enero del año siguiente.

Recordaba Ocaña que el Governador no pot entrometres en causes y negocis de la Cort de la Batlia, ni en lo provehit y sentenciat per aquell, conforme al privilegi 7. del R. en Ferrando. 2. (74); cita sentencia, posterior, de 14-X-1587, inserta in libro provisionum et litterarum Regiarum Baiuliae generalis Oriolae fol. 450 , pág. 2 (74, v.). A favor del Baile sentenció la Real Audiencia de Valencia en $28-I X-1588$, en cierto pleito sobre pago del almoxarif por el Cabildo de Orihuela, erigido en 1566, por desmembración del de Cartagena (106, v.).

La Bailía tuvo siempre cuidado de formar colecciones legales, de sus atribuciones, como el Llibre intitulat de diferents privilegis y ordens reals del Rey D'Aragó, cuyo Índice publicó $M^{a}$ Milagros Cárcel Ortiz en "Ligarzas", 7 (1975), así como en la 2, de 1970, había dado $M^{a}$ Desamparados Cabanes Pecourt El Llibre Negre del Archivo General del Reino de Valencia, rúbricas referentes a Oriola, como la Provisio entre los batles generals de Regne de Valencia e de Sexona enlla sobre la responsio dels dotze milia sous per les alcaydies dels castells de Oriola e Alaquant.

Especifica la Declaracio del Capitol $x$ de los traus ques fan no pagant dret de saca de Castella, vol que no portant albara de haverlos pagat en Castella se pague açi dret Real de Almoxarif. Estaba establecido que Los qui entraran roba de Castella sense albara del Desmero, son tenguts de pagar lo dret de Almoxarif en aquesta ciutat exceptades les coses declarades en lo $n .2$ y mes deu pagar: lo dret de venda que fara de tal cosa $(69, \mathrm{v} .70, \mathrm{r}$.$) .$ 
Ya en el Capitol II se estableció que si algu entrara en terme de la present ciutat, mercaduria alguna de Castella, ans de fira, la qual roba y mercaduria, com es notori, deu lo dret de entrada a la Quema, tambe al dret de Almoxarif, no portant albara del Desmero, la pena en que incurria; distinguíase entre fira y mercat, aquélla era por privilegio real como el de Alfonso $X$ de Castilla de $3-V-1272$ (40, v.-41, r.).

Ocaña exponía con el mayor celo la legalidad de la exacción del dret Real del amoxarif, rebatiendo las alegaciones por exención, de acuerdo con la tradición del impuesto. Es de notar la coetaneidad de los textos de Fr. Miguel Salon, O.S.A. (1539?-1621), que en 1587-1590 dictaba en la Universidad de Valencia los cursos de lusttitia et lure y su Disputatio de tributis vectigalibus, como su maestro Juan Blas Navarro había publicado en 1587 De Vectigalibus. Distinguía Salón en Castilla la Décima real; la terciodécima o tercera parte de los diezmos; el portazgo y la alcavala; y en el Reino de Valencia los tributos pagados al rey, peaje, quema, quinto del pescado y diezmo de mar; los de la Diputacio del General, el general, el novum impositum o dret nou, y el dret Real de la Sal; y los tributos pagados a la ciudad bajo el nombre genérico de sises, todo ello estudiado en 1972 por José Aliaga Girbés en Los tributos e impuestos valencianos en el siglo XVI. Su justicia y moralidad según Fr. Miguel Bartolomé Salón, O.S.A. (1539?-1621), editado por el Instituto Español de Historia Eclesiástica, de Roma.

En el Capitol XXIIII del Llibre, de Ocaña, se decía: Nota que aquest dret com los demes drets Reals se acostuman arrendar de tres en tres anys com està axi dispost per dret comu dels Romans, aduciendo textos jurídicos y concluyendo que lo costum de aquesta ciutat y provincia es axi, por Pascua de la Natividad, y luego se hacian en la ciutat de Alacant, para los que se arrendaban en ella.

En 1602 se había publicado el Memorial tret per Mossen Gaspar Ocaña, Credencier de sa Magestat del Peatge y Quema y altres drets Reals de la present Ciutat y regne de Valencia, del que han de pagar les mercaderies als drets del Peatge y Lleuda; aixi com de les que per mar vindran o passaran; fue mandado imprimir por el Baile general de Valencia don Gaspar Mercader, junt al Moli de Rivelle; cítalo José RibeIles Comín en su Bibliografía de la Lengua Valenciana (III, 126); el Llibre de Luis de Ocaña, de 1613, se hallaba, pues, en aquella línea.

En 7-III-1604 se concedía desde Alcalá el privilegi del dia franch dado por Felipe III; otorgaba que la ciudad tuviese un día franco de pago de todos los derechos reales. En 1609 actuaba, como tradicionalmente, la Junta Patrimonial de la Ciutat y provincia de Oriola (72 v.73 r.). 
En 1611, según Ocaña, totes les Universitats y llochs de les hortes de Oriola, no tenen ni gozen de different franquea, si no es de la mateixa que Oriola; y los Jurats de Oriola donen les franquees a tots los que vihuen en dits llochs de Redova, Coix, Granja, Albatera, Catral, Daya, Guardamar y Benejuzer y Rojals, e si Callosa y Almoradi per se Universitats, donen tranquees als habitants en dites Universitats $(87, v$.$) .$ Terminaba Ocaña el Capitol XXI escribiendo: Lo dret del Almoxarif se ha de cobrar y jutjar conforme capitols, y faltant capitols, segons se haura usar, practicat, y jutjat, y la raho n. 2.

En diciembre de 1611 era Portantveus de general Governador del Regne de Valencia dellà Xixona Don loan Ferrer (62, v.-63 r.). Ocaña iba explicitando en su Llibre las declaraciones de cada Capitol; en el X trató de los drets de saca de Castella y de los franchs, del Delmero de Castella.

En cuanto a los cambios de avecinamiento al pasar de un reino a otro el Capitol XV aclaraba que «En lo mudar domicili y passar sa casa se aquella ciutat y Regne al de Castella qualsevol vehi ò estrany, es tengut a pagar dos reals al Collector ò Arrendado del dit dret Real de Almoxarif, $y$ estos dos reals son Valencians de a diguit dines cascun que fan tres sous y no dos reals castellans que son tres sous y deu diners» $(73, v$.), o sea, dos dihuytens, de 18 dineros, que hacia cada uno sueldo y medio, sou y mig, circulantes en aquellos años, 1613, siendo la moneda valenciana la propia de aquesta Ciutat y Provincia, como se designaba a Orihuela y su Gobernación, las sentencias de cuyo Batle no se apelaban, por otra parte, a la Real Audiencia de Valencia sino al Supremo Real Consell de Aragó.

En el Capitol XX se trata del crim de salvateria, aduciendo de nuevo el derecho romano: En aquest capitol, se posen y declaren les penes en que es encorregut $y$ deu ser condemnat lo qui cometrà crim de Salvateria lo qual se comet, comprant home franch en son nom y franquea per a home pecher, a si de excusarlo de pagar los drets Reals. E tambe es comet fent compania ab pecher, despachant en son nom merdauria del tal pecher o, foraster, com lo dispon la .1.7 tit-25 del Almoxarif de Carthagena y Murcia, lib. 9, nov. recop. fol. 320, refiriéndose a la Nueva Recopilación, y añade: Aquest crim de dret comu de Romans, apellatur de lictum excusationis, et civile, licet criminaliter, intentetur $(88, v$. 89, r.).

Se especificaba perque de dret comu dels Romans semblant dret no es degut es exegeix si no es de bens mobles que sunt in commertio; recoreando las gabelas y el privilegi del dia que la Magestat del senor Rey don Phelip 3 concedi a la present ciutat de Oriola sa data en la vila 
de Alcala a 7 de Mars 160 (7, r.); distinguía entre los que eran franchs y los pechers; remitiendo a la Nueva Recopilación $(7, v$.).

En la Declaració del Capitol XXV, último del almoxarif, termina considerando el dan que los Arrendadors de drets Reals, y los Escrivans de les Corts tingueren en occasio de la, expulsió de los Moriscos del present Regne, que la Majestat del Rey Phelip 3 nostre Senñor, manà fer ab son Real bando, publicat en la ciutat de Valencia en 22. Setembre. $1609, y$ en la ciutat de Oriola a 26. de setembre de dit any se donaren diverses sentencies de refactio ( $111 \mathrm{v}$. $112 \mathrm{r}$.), la raho la refactio en la doctrina de Bart. in. 1. Cotem. ferro qui maximos n. 4 de publ. et vectig.

En 1614 Francisco Álvarez publicó el Tratado contra el Libro titulado Almojarifazgo que compuso el Doctor Luis de Ocaña, sin que ahora se entre en aquél, dado el propósito de utilizar sólo el aquí enunciado.

\section{H.-EL DRET DEL VEDAT}

El segundo dret que expone Ocaña está en los Capitols ab los quals se arrenda el dret Real appellat del Vedat, en les ciutats de Oriola y Alcant, viles Elig, Crevillent, y Val de Elda y en tota la Batlia general deça Xixona (113).

Únicamente podía arrendarlo el Baile general d'enllà Xixona, en Orihuela primero, luego en Alicante; y se arrenda tambe o es dona a collectar en les Taules de Elig, y Crevillent, y de tota la Vall de Elda, en la qual Vall entra Asp, Novelda, Elda, Petrel, Monover, y les Salines $(115, v$.$) .$

Este dret, extractionis rerum prohibitarum, venía regulado por el privilegio de Alfonso III de Valencia, de 6-X-1448, que mandaba que en ello conociera siempre el Baile general y non per deputatos generalis regno; como está en el Aureum Opus, XVII, CCXXXIX v.; así como por el Pedro II, de ix kal. marcij A.d.M.ccc. xliij, que disponía Quod iurati non se intromitant super extractione rerum ac super alijs patrimonium regium tangentibus et officio baiulie pertientibus, que se halla en el Aureum Opus, c. XLVII, fol. 115. Ocaña inserta una disposición de 1573; Aquesta Real Sentencia com les demes que donen en lo S.S. Consell de Arago, fan ley en tot lo regne (118 v.).

Estaba prohibida - vedada - la extracción de pez, sebo, alquitrán, madera, cáñamo, hilo de jarcia, hierro, armas, caballos, trigos, cevadas, ganados, oro y plata, monedado y no monedado, arroz, daça, panizo, y otros por los mismos fueros, privilegios y pragmaticas del nuestro 
Reyno de Valençia prohibidas; se recordaba en la Real Pragmática de 24-XII-1599 (119-122), y otra de 7-IX-1610 que fijaba la pena pecuniaria por la infracción, en cinquenta libras moneda de Valencia (123, v.-124 r.).

El Aranzel del dret Real del Vedat que inserta Ocaña (129, v.-134, r.) es una preciosa pieza para el estudio de la lengua valenciana de la época, sin olvidar aquí su valor económico; pero hallándose impreso, se brinda a los filólogos más que a los economistas, por poco divulgado.

\section{III.-DRET DEL REAL SERVICIO Y MONTAZGO}

Así lo titulan los Capitols ab los qual se arrenda y collecta lo dret Real del servicio y Montazgo en la ciutat y Batlia general de Oriola, de Xixona a esta part $(136, \mathrm{r}$.$) .$

Se exigía el uno por ciento de las reses: de cent cabres, una; de cent porchs un porch; de cent borregos un borrego; el arrendador o comprador de dit dret, sia tengut pagar lo preu en dos iguals pagues, ço es la primera a Tots Sants, y la segona, pera Carnestoltes.

Se enumeran los cargos al servicio de la colecta de este dret: collector, fieler, corredor, macer, verguer y escriva. Se establece que Lo dret de servici y Montazgo es Regalia de la Majestat. Lo de servici y Montazgo no se arren a ni collecten en altra part de tot lo Regne, si no es en la Ciutat y Batlia general de Oriola. Lo dret de servici y Montazgo, resta en la ciutat y Provincia de Oriola del temps que era dels Reys de Castella. La Ciutat y Provincia de Oriola era dels Reys de Castella, y per sentencia arbitral se adiudica al Rey de Valencia. La Ciutat y Provincia de Oriola, usa de costums y privilegis contraris als furs y la raho ibi. Lo Rey en laume segon uni dita Ciutat y Provincia de Oriola al Regne de Valencia. Lo Batle general de Oriola y no altre arrenda y nomena Fieler $y$ coneix de dit dret de servici. Lo Rey nostre señor mana al Batle de Oriola posas remey enque los Cabañers Castellans no defraudassen dit dret; éstos son los puntos de la Rúbrica (138, v.-139, r.). La última disposición que cita Ocaña es la del Marqués de Aytona, virrey de Valencia, de 15-VI-1591.

\section{IV.-DRET DEL MIG DELME}

En fol. 143 los Capitols ab los quals se arrenda y collecta lo dret de 
Mig Delme appellat del Estremeño, en la ciutat y Batlia general de Oriola, de Xixona a esta part disponian que todo ganado que entrase a pacer en el territorio de la Bailia, fuese Castilla, de Aragón o del Reino, había de entregar de cada cincuenta corderos nacidos en la demarcación, uno, en la proporción que se detalla, entre machos y hembras. Se establecía que Lo dret de Estremeño, o Mig Delme, es ragalía de sa Majestat; persona alguna forastera estaba exenta del mismo, menos los vehins $y$ domiciliats en la present Batlia, que no pagaban tal dret; la razón era que ya pagaban el delme o diezmo y los forasteros sólo satisfacían la mitad, el mig delme, por entrar sus ganados.

Explicitaba Ocaña la diferencia entre el Delme del carnatje, y el dret de Estremeño o de mig Delme; se aducía el privilegio de Fernando ll de 11-XII-1482, De potestate ac superioritatibus baiuli generalis, VII del mismo en Aureum Opus, folio CCXV; e inserta modelo de certificatoria o albarà del fielero, sobre corderos nacidos en su territorio, que era el del Bisbat, Governacio y Batlia general de Oriola (146, v.-147, r.). De Leopoldo Piles Ros es el libro titulado Estudio documental sobre el Bayle general de Valencia. Su autoridad y jurisdicción (I.A.M., 1970), donde se citan documentos referentes a Oriola de 1424, 1427, 1467, referentes a paso de moros y el de 24-III-1431 del Baile general encargando a Johan de Masquefa el cobro de los bienes que cita pertenecientes a castellanos, con utilización del Capitulos con los cuales se arrienda y colecta el derecho Real de Almoxarifazgo de la Ciudad de Orihuela y Valle de Elda, en su edición en castellano.

\section{V.-DRET DE PASTURA}

En folio 150 comienzan los Capitols ab los quals se arrenden los herbes y dret de pastura, de les redondes que te sa Majestat en los camps de la present Ciutat de Oriola, en les partides appellades de la Mata, Genibralet, Benijofer, Molar y camp del terme de Almoradi.

Especifica lo dispuesto sobre alguns ganados forasters, entrados a herbejar, cuyo amo eia tengut a pagar y pague lo preu de dita herba, a sola instancia del Procurador Patrimonial de sa Majestat, fahedora davant lo Batle.

Se recordaba que Aquest dret de Pastura, es regalia de sa Majestat, com apar per privilegi 5 Regis Alfonsi 3, fol. 176, esto es, de Alfonso III de Valencia, en el Aureum Opus, aquel folio; Promittit dominus rex quod non alienabit loca bona et iura hic expressa, de 29-I-1418. Felipe II hizo gracia a la ciudad de Orihuela de la partida del camp dela Matan- 
ça, la partida del camp deles Salines, y la partida del camp del Alcachofar y Pergamisa, y Rabat (151).

Se permitía a los Cabaners Arrendadors de dites redondes llevar a pasturar a sus ganados a lo Realench, que era desde los Alcors a la Mar, y del port de la Bojosa y de la rambla del Pi, y de la Lloma del cap de Serve a la Mar (152, v.-152, r.).

\section{VI.-DRET REAL DELS PESOS DEL PEIX}

Al folio 153 los Capitols ab los quals se arrenda en la present Ciutat lo dret Real dels pesos del peix.

Se establecía que qualsevol traginer o qualsevol persona que a la present Ciutat portara a vendre peix del mar o albuferes de Castella, o de altra part del present Regne, sia tengut de venir nec avia a la Peixcateria ò plaça de la present Ciutat y pesar y arromanar lo tal peix, en lo pes major de dita Peixcateria, y luego venderlo públicamente en dicha plaza, pudiendo así los arrendadores y colectores los derechos de Almoxarif y Quema exigir lo pertinente a ellos. Se establecía que del Riu, ò Assequies ò Asarps que sea peixcat ab caña, ò ab qualsevol altre art ò enginy, no es puixa cobrar ni es pague lo dit dret. Se añadía que el dret dels pesos del Peix, es regalia de sa Majestat, com apar per furs y privilegis del present Regne, com a tal regalia fonch incorporat a la Corona y Real Patrimoni $(154$, v.); recuerda el forum 1, Rubrica 25 . de offici de pes, libro 9 fororum.

\section{VII.-DRET REAL DE ADUANA}

Al folio 156 los Capitols ab los quals se arrenda y collecta lo Dret Real de Aduana, en la Ciutat e Alacant, Universitats de Monfort, Muchamel y S. Ioan, y Benimagrell, y los termes generals de dita Ciutat.

Se estableció que ninguna persona de qualsevol estat y condicio sia, axi vehi com estrany, no sia osat carregar ni descarregar robes ni mercaduries algunes en lo lloch nou y terme de la vila de Elig, ni en terme general de la present Ciutat fora moll, sens expressa llicencia del Batle general de dit dret $(158, \mathrm{r}$.$) .$

El apartado 18 de este capitulo establecia que qualsevol que portas per mar qualsevol catiu, negre, o blanch axi home com dona, que o sia batejat, sia tengut a pagar per dret de cabeçaje quaranta diners per cada cap, y aquestos sia obligat pagar en ser surta la nau, o navili en lo 
present port, y si seran batejats, no sien tenguts a pagar lo dit dret de cabeçaje $(159, \mathrm{v}$.$) .$

Todo el que no gozaba de franquea, esto es, estaba exento por alguna razón, venía obligado a pagar al Duaner el dret Real de Aduana, com a pecher. Pecher es dit tot hom que no tendra privilegi de franquea de dit dret de Aduana (163, r.).

Dice el Llibre de Ocaña: in verbum Aduana: Aquest nom y paraula Aduana, es Arabiga y significa casa señalada, ò designada, per los lusticia y lurats y Consell de la Ciutat, vila, ò loch hon se porten a manifestar y despachar totes les robes y mercaduries que dehuen dret $(163, \mathrm{v}$.). Pagaba lo venedor per dret de venda deu dines per lliura de moneda, $y$ lo comprador cinch dines per lliura, si los dos seran pechers.

Hace referencia al libro 9 de la Nueva Recopilación, Almojarifazgo del Arzobispado de Sevilla y a los de Cartagena y Murcia; expone una causa posada per Gines Miralles, Notari, Procurador Fiscal Patrimonial de sa Majestat contra un mercader genovés que no había obtenido licencia para cargar y descargar dos Sageties de vi que portaren de Benicarlo per a vendre en la vila de Elig sens procedir dita llicencia del Batle general, con sentencia del Supremo Consell de Aragó, de 3VIII-1519; es todo un documentado repertorio de gran interés para el Derecho mercantil y marítimo en particular.

Termina la exposición del dret de Aduana con un privilegio de Felipe II de 21-XII-1591 creando la función de un fiel o credençer entre los tractants y arredadors $(176, r$.).

\section{VIII.-TTRETA Y VENDA DE LA SAL}

Los folios 178 a 185 del Llibre de Ocaña están dedicados a los Capitols ab los quals se arrenda la treta y venda de la Sal, de les Salines de la Mata, que estan en terme de la Ciutat de Oriola. Éstas tenian casa Almazen construida por orden de Su Majestad en la ciudad e Alicante; se embarcaba para Nápoles mediante provisión del Virrey o lutge de la Sal de la Ciutat de Napols. Se especificaba que La sal y les Salines, y lo dret de arrendar y vendra aquella, es regalia de sa Majestat. Lo fer capitols pera subhastar y arrendar les Salines, se esguarda al Batle general y demes officials patrimonials de la junta patrimonial, privative ad alios $(181, \mathrm{r}$.). Se recordaba que Juan I de Aragón en 28VII-1389 dio licencia a la ciutat de Oriola per a poder fer albufera en les Salines de cap de Serva, y que en 1594 el Procurador Patrimonial 
mandó tancar la boca de la Albufera de Oriola, pero lo dany que rebia de les Salines de la Mata. Lo señor Rey está en possessio de vendre la sal de les Salines de la Mata, franca del dret del General.

Recordaba el privilegio 5 de Alfonso III, folio 176, el. CLXXVI del Aureum Opusque non alienabit loca et iura hic expressa, ya citado. Se acostumbraba a encargar al Batle de Guardamar que mane a la gent del poble que vaja a traure, ò, a carregar sal y que vinga a asistir al carregar.

Finalmente, se especificaba que per qualsevol nao que carregara de les parts de Llevant deu pagar quatre ducats, al Administrador ò al Batle, o su representante; dela part de Ponent dos ducats: de carregar una Pollaca, set reals; de una Sagetia onze reals, de cada Barca que carregara sal pera a les gavelles del Regne, quatre reals de expedicio fora del dret que li tocara de carregar ab la gent. Finalmente, se hacia constar que Les Salines de la Mata eo de Guardamar alias appellades del cap de Server, son de sa Majestat, y les altres salines que estan en dita partida del cap de Server deves Ponent, son de la Ciutat de Oriola, como constaba por privilegios de Sancho IV, de Castilla, de Pedro IV de Aragón - Il en Valencia- de 1364 y de Juan I de Aragón de 1389.

Termina el Llibre con la disposición de Felipe II de 18-I-1590 no accediendo a la pretensión de los Diputados de la Generalidad sobre exacción del derecho de la sal, por conservar la possession en que esta mi Regia Corte de vender la dicha sal.

El folio 185 tiene como colofón Finis. Tractatus de Regiis redditibus in Baiulia generalis Civitatis Oriolae et Alicantis locandis et exigendis.

El Llibre de Luis de Ocaña está en la línea que seguirian otros autores, como Vicente Branchat, Tratado de los derechos y regalias que corresponden al Real Patrimonio en el Reyno de Valencia y de la Jurisdiccion del Intendente como Subrogado en lugar del Bayle general (1784-61, 3 vol.), que sirvió en la administración de los años siguientes a la Nueva Planta. 